\title{
Délibérations du Comité central de la FMH
}

FL. Lors de sa séance du 27 janvier 2000, le Comité central (CC) a traité, entre autres, des affaires suivantes:

\section{Assurances sociales}

1. Financement du CSPV - Ajournement de la mise en vigueur de la loi sur les produits thérapeutiques

La Chambre médicale de juin 1999 a décidé de continuer à assurer son soutien au CSPV (Centre suisse de pharmacovigilance) en lui allouant pour l'an 2000 une contribution spéciale de 5 francs par membre FMH (des catégories 1, 2, 3 et 4), cela afin de préserver l'existence du CSPV tout au moins jusqu'à la création d'une nouvelle réglementation dans le cadre de la loi sur les produits thérapeutiques. Dès lors que la date prévue pour l'entrée en vigueur de cette loi ne correspond pas aux vœux du DFI, il ne faut pas compter que la nouvelle réglementation sur le recensement des effets indésirables de médicaments voie le jour avant la fin 2001.

Or, vu la faveur dont jouit le CSPV auprès du corps médical, le CC consultera la Chambre médicale ordinaire de juin 2000, sur la poursuite ou non en 2001 de la contribution spéciale à l'égard du CSPV.

2. Loi sur les produits thérapeutiques (LPT) appréciation après consultation de la Commission du Conseil national pour la sécurité sociale et la santé publique (CSSS-CN) - démarches futures de la FMH.

Après avoir été examiné par la CSSS-CN, ce projet de loi passera devant le Conseil national lors de la session de mars 2000. Le CC se penche essentiellement sur les modifications apportées par la CSSS-CN aux dispositions concernant la compétence de distribution, la vente par correspondance, la préparation et le stockage des produits thérapeutiques et sur les recommandations concernant la prescription et la distribution des médicaments, lesquelles, selon l'avis de la commission, doivent être édictées par l'Institut suisse des produits thérapeutiques.

Selon le vœu du CC, un résumé de la discussion sur les thèmes précités est publié dans cette édition du Bulletin des médecins suisses (pages 496-497), un papier sera rédigé à l'intention des parlementaires et, à propos des produits thérapeutiques, contact sera pris au plus haut niveau avec la Société suisse de pharmacie.

\section{Assurance qualité}

Dénonciation du contrat QUALAB pour modification

La manière de procéder des assureurs en matière de sanctions contre les détenteurs de laboratoires négligents a montré qu'à cet égard les dispositions du contrat QUALAB ne sont plus adaptées à la situation actuelle (nouvelle loi sur l'assurance-maladie [LAMal] et surtout conventions bilatérales relatives à la structure tarifaire TarMed), cela d'autant plus que la Liste des analyses fait aujourd'hui partie intégrante de la structure tarifaire. De plus, la FMH est d'avis que la commission QUALAB ne peut pas être juge et partie, à savoir à la fois édicter des critères, des standards et des dispositions concernant les objectifs et exercer une fonction de juge en sanctionnant le non-respect de ces prescriptions.

Le CC a donc décidé de dénoncer le contrat pour modification afin qu'à l'avenir les sanctions à l'encontre des médecins en pratique privée soient fixées et appliquées dans le cadre des conventions passées entre le CAMS et la FMH, d'une part, et la CTM/AM/AI et la FMH, d'autre part.

\section{Prévention}

Campagne nationale de prévention contre l'alcoolisme "Ca débouche sur quoi?»; sous-projet concernant les médecins: formation continue des médecins de premier recours.

Conformément à une décision antérieure du CC, le département de la prévention de la FMH sera associé à ce projet (pour la partie "médecins») lancé par l'Office fédéral de la santé publique (OFSP), conjointement avec l'Institut suisse de prévention de l'alcoolisme et autres toxicomanies (ISPA) et la Régie fédérale des alcools. D'accord avec le projet de cours qui lui est présenté, le CC décide de les placer sous l'égide de la FMH et de leur assurer l'utilisation du logo de cette dernière. La recherche de parrainages de ces cours est laissée à la direction générale du projet, la FMH ne souhaitant pas s'y associer. En revanche, elle entend participer activement aux évaluations. 


\section{Formation postgraduée et continue}

1. Révisions des programmes de formation postgraduée sur Internet (www.fmh.ch)

Avec l'entrée en vigueur probable des accords bilatéraux avec l'UE en 2001 et simultanément de la loi sur les professions médicales, basée sur ces accords, des nouvelles conditions-cadres seront créées qui exigeront, notamment lors de la création ou de la révision de programmes de formation postgraduée, davantage d'informations et de possibilités de collaboration de la part des médecins concernés. Le CC, au sens d'une mesure immédiate, a donc résolu de rendre accessibles sur Internet tous les programmes actuellement en révision. Dans un souci de transparence et de formation d'opinion, le CC estime en effet qu'avant la création de nouvelles spécialités, la prolongation des cursus de formation ou la prescription d'exigences supplémentaires en matière de formation postgraduée, il serait judicieux que les intéressés puissent s'exprimer. Toutefois, la maittrise des travaux administratifs comme le développement rationnel de ces moyens impliquent certaines règles à respecter. Les présidentes et présidents des sociétés de discipline médicale seront prochainement informés de cette décision et de la nature de ces dispositions qui seront déjà applicables aux révisions et nouveaux programmes examinés par la CFPC durant le deuxième semestre de cette année.

\section{Organisation}

1. Création d'une fondation suisse pour l'encouragement à l'allaitement

L'OFSP prévoit la création de cette fondation en collaboration avec le Comité suisse pour l'UNICEF et la Fondation suisse pour la promotion de la santé (Fondation 19) en vue d'établir des structures appropriées à cette future collaboration et d'assurer le financement de l'encouragement à l'allaitement en Suisse.

Le CC décide de s'associer à la création de cette fondation et de participer aux frais généraux en lui allouant de 2001 à 2003 un montant annuel de 10000 francs. La FMH sera représentée au conseil de fondation par le Dr Ursula Steiner (membre du CC).

\section{Cours de formation postgraduée pour experts} médicaux dans le domaine de l'assuranceaccidents.

Après le succès remporté par les cours qui ont eu lieu l'année dernière à Lucerne, il convenait que les médecins de langue française aient également la possibilité de suivre de tels cours. Ceux-ci seront donnés les 25 et 26 mai 2000 à Montreux sous la direction du Prof. R. Darioli, qui entend toutefois limiter sa collaboration à la Romandie. Le CC approuve l'organisation de ce cours par la FMH, sous le patronage de la Société suisse de traumatologie et de médecine des assurances et de l'Union suisse des sociétés chirurgicales, avec la collaboration du département de la médecine des accidents de la SUVA et du service médical de l'Association suisse des assurances.

\section{Surveillant des prix: affirmations sans substances}

(FMH) Lors de sa conférence de presse du 24 février 2000, le surveillant des prix a déclaré que la structure tarifaire TarMed produirait une augmentation des coûts de 300 millions de francs. Il s'agit là d'une affirmation dénuée de tout fondement rationnel. La moindre estimation des coûts tarifaires demanderait, en effet, que les valeurs du point tarifaire soient fixées. Ce qui n'est le cas pour aucun des futurs tarifs.

La FMH déplore l'attitude du surveillant des prix, qui a une fois de plus tenté, par ses affirmations sans substance et tendancieuses, d'entraver l'introduction des nouveaux tarifs. 